\title{
Lobomicose e tratamento cirúrgico: um relato de caso no interior da Amazônia
}

\author{
Lobomycosis and surgical treatment: a case report in Amazon countryside
}

Lobomicosis y tratamiento quirúrgico: informe de un caso dentro de la Amazonía

\begin{abstract}
Elizângela Willott Pereira ${ }^{1 *}$, Jennyfer Conceição dos Santos Monteiro ${ }^{1}$, Flávio Mileo Bacelar Guerreiro?2.
\end{abstract}

\section{RESUMO}

Objetivo: Relatar um caso de Lobomicose atendido no município de Monte Alegre-Pará, interior da Amazônia. Relato de experiência: Paciente masculino, com 35 anos de idade, apresentava lesão queloidiforme em membro inferior esquerdo com 3 anos de evolução, com diagnóstico prévio de Lobomicose por exame histopatológico e tratamento anterior com criocirurgia, porém, com recidiva da lesão há um ano. Foi proposta uma nova tentativa de tratamento por exérese da lesão, sem sinais de recidiva há 18 meses. Considerações finais: Como a lobomicose é vista em áreas localizadas e, em geral, pouco desenvolvidas, há uma falha substancial de conhecimento científico em relação ao assunto; isso justifica a importância do estudo frente a esse cenário. Além disso, até o momento, na literatura, não foi apresentada terapia inteiramente eficaz contra o agente Lacazia loboi. No relato do caso, no entanto, o paciente apresentou resposta completamente satisfatória com a terapia empregada.

Palavras-chave: Lobomicose, Lacazia, Dermatomicoses.

\begin{abstract}
Objective: To report a case of Lobomycosis treated in the city of Monte Alegre-Pará, in Amazon countryside. Case report: Male patient, with 35 years old, had a keloid injury in the left lower limb with 3 years of evolution, with previous diagnosis of Lobomycosis by histopathological examination and previous treatment with cryosurgery, however, with recurrence of the lesion a year ago. A new attempt at treatment due to excision of the lesion has been proposed, without signs of recurrence for 18 months. Final considerations: As lobomycosisis seen in specific areas and, in general, poorly developed, there is a substantial lack of scientific knowledge on the subject; this justifies the study's importance in this scenario. In addition, so far, at literature, no fully effective therapy against the agent Lacazia loboi has been presented. In the case report, however, the patient showed a completely satisfactory response with the therapy applied.
\end{abstract}

Keywords: Lobomycosis, Lacaziai, Dermatomycoses.

\section{RESUMEN}

Objetivo: Reportar un caso de lobomicosis tratado en el municipio de Monte Alegre-Pará, interior de la Amazonía. Informe de experiencia: El paciente masculino, de 35 años, tenía una lesión queloidiforme en la extremidad inferior izquierda con 3 años de evolución, con diagnóstico previo de lobomicosis por examen histopatológico y tratamiento previo con criocirugía, sin embargo, con recurrencia de la lesión hace un año. Se propuso un nuevo intento de tratamiento debido a la escisión de la lesión, sin signos de recurrencia durante 18 meses. Consideraciones finales: Como la lobomicosis se observa en áreas localizadas y, en general, poco desarrollada, existe una falta sustancial de conocimiento científico en relación con el tema; Esto justifica la importancia del estudio en este escenario. Además, hasta ahora, en la literatura, no se ha presentado una terapia totalmente efectiva contra el agente Lacazia loboi. Sin embargo, en el informe del caso, el paciente mostró una respuesta completamente satisfactoria con la terapia empleada.

Palabras clave: Lobomicosis, Lacazia, Dermatomicosis.

1Universidade do Estado do Pará (UEPA), Santarém - PA. *E-mail: elizangela.willott@gmail.com

2Universidade Federal do Rio de Janeiro (UFRJ), Rio de Janeiro - RJ. 


\section{INTRODUÇÃO}

A Lobomicose foi descrita pela primeira vez por Jorge Lobo em 1930 e se caracteriza por uma infecção fúngica granulomatosa crônica que afeta tecidos cutâneo e subcutâneo. Essa patologia é causada por um fungo saprófita, Lacazia loboi, classificado no grupo da ordem Onygenales e da família Ajellomycetaceae, que vive em solo, vegetação e água em áreas de floresta quente e úmida com rios. A lobomicose caracteriza-se por lesões nodulares ou queloides (BELTRAME A, et al., 2017).

As pessoas mais atingidas pela enfermidade são trabalhadores rurais nas regiões em que predomina as características de vegetação descritas, como América Central e América do Sul. A única forma conhecida de transmissão do parasita é a inoculação do fungo. Nunca foi descrita a transmissão inter-humana da doença (KORTE RL, et al., 2019). Um grande desafio que envolve essa patologia é o tratamento. A terapêutica varia de acordo com localização e extensão das lesões cutâneas. Entretanto, não existe até o momento nenhuma terapia descrita na literatura totalmente eficaz e livre de recorrências (FURTADO NA, et al., 2013).

Portanto, como essa enfermidade é vista somente em regiões localizadas e em áreas, em geral, pouco desenvolvidas, há uma falha substancial de conhecimento científico e, com isso, no tratamento específico e eficiente para a Lobomicose (SOUZA MN, et al., 2015). O objetivo do estudo é relatar um caso clínico de um paciente do sexo masculino com diagnóstico de Lobomicose ou Doença de Jorge Lobo atendido no município de Monte Alegre, estado do Pará.

\section{DETALHAMENTO DO CASO}

Paciente, 35 anos de idade, sexo masculino, natural e residente do município de Monte Alegre/PA, solteiro, atualmente trabalha como pedreiro. Trabalhou por 10 anos na agricultura. Foi atendido em novembro de 2018. Compareceu à consulta com histórico de lesão tumoral de aspecto queloidiforme com bordas definidas e descamação furfurácea de aproximadamente $4 \mathrm{~cm} \times 3,5 \mathrm{~cm}$ em face medial do joelho esquerdo, com 3 anos de evolução (Figura 1). Negava prurido ou ardor. Trouxe resultado de exame histopatológico com diagnóstico de lobomicose. O paciente foi submetido ao tratamento em outro nosocômio, onde foi realizada criocirugia com nitrogênio líquido, o quadro evoluiu com piora progressiva, representada por recidiva em cerca de um ano.

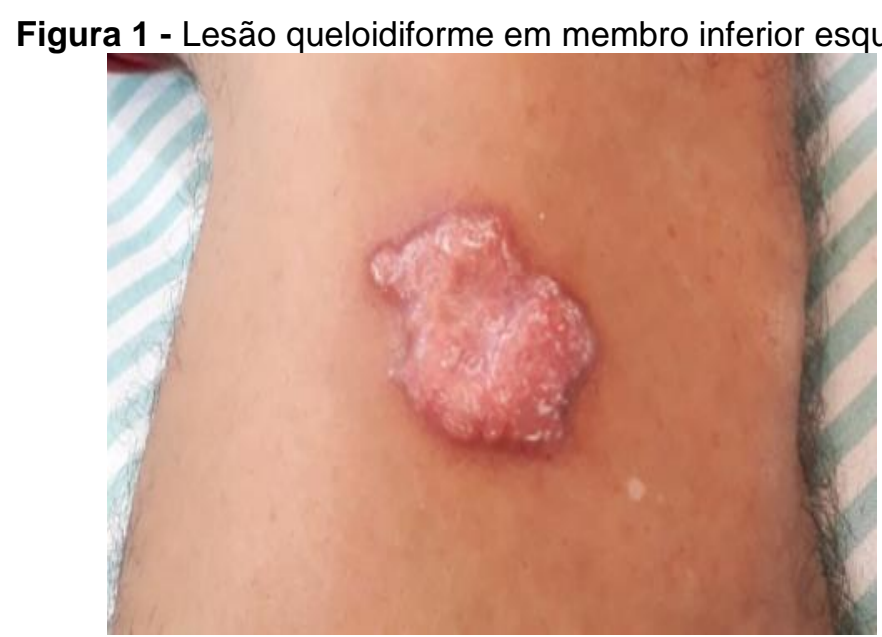

Fonte: Pereira EW, et al., 2020.

Em janeiro de 2019 foi proposta uma nova tentativa de tratamento. Por ser uma doença localizada, foi optado pelo tratamento cirúrgico com exérese da lesão com margem de $4 \mathrm{~mm}$ até o tecido subcutâneo e reconstrução com retalho em rotação de pele de panturrilha (Figuras 2 e 3). Não foram administradas medicações antifúngicas orais ou tópicas. Os pontos cirúrgicos foram mantidos por 21 dias e realizados curativos locais com vaselina. Paciente está em acompanhamento há 18 meses sem sinais de recidiva (Figura 4). 
Figura 2 - Exérese da lesão.

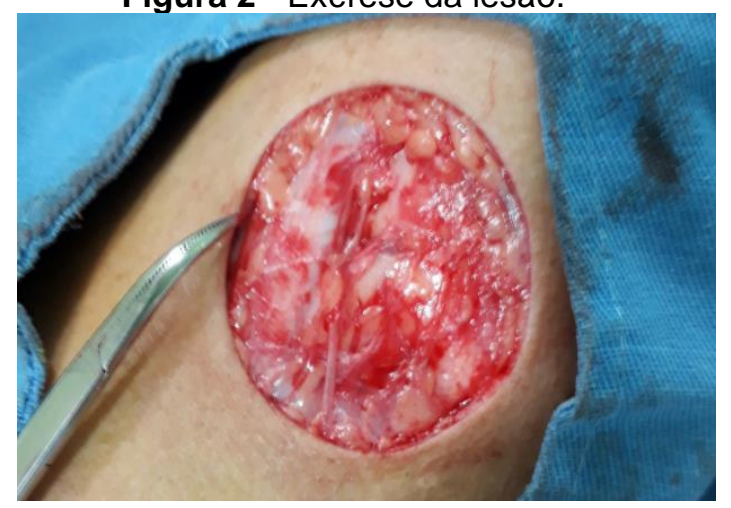

Fonte: Pereira EW, et al., 2020.
Figura 3 - Pós-operatório.

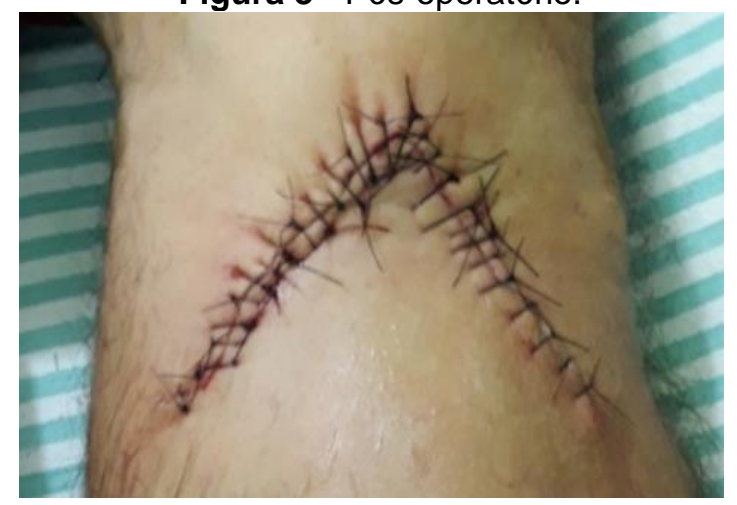

Fonte: Pereira EW, et al., 2020.

Figura 4 - Dezoito meses após a cirurgia.

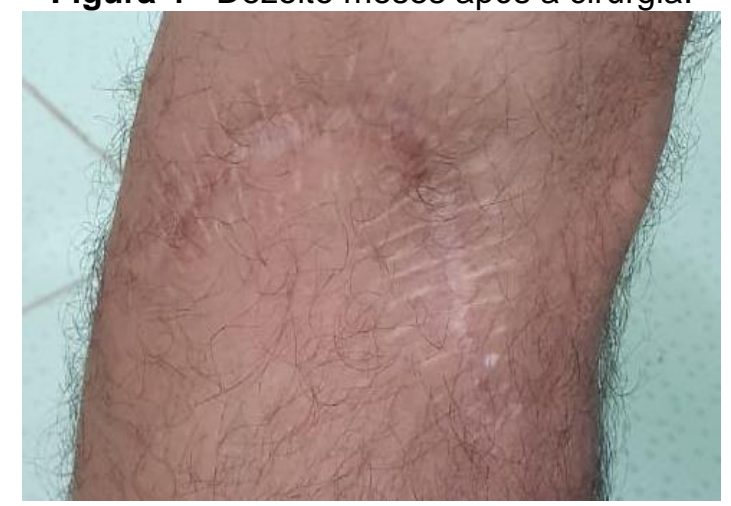

Fonte: Pereira EW, et al., 2020.

\section{DISCUSSÃO}

A Lobomicose ou Doença de Jorge Lobo recebeu inúmeros sinônimos desde a sua descoberta: lacaziose, blastomicose queloidal, blastomicose tipo Jorge Lobo, lepra do caiabi, granulomatose blastocoide, blastomicose da Amazônia (TAVARES R, et al., 2011). Além disso, antes da consagração do fungo como Lacazia loboi, ele recebeu outras denominações como: Glenosporella loboi, Blastomyces brasiliensis, Glenosporopsis amazonica, Loboa loboi e Paracoccidioides loboi (RIVITTI EA, 2018).

Os casos da doença ocorrem principalmente em regiões tropicais e subtropicais, de clima quente e úmido, com abundantes cursos de água e índice pluviométrico superior a $2.000 \mathrm{~mm}$ anuais e temperatura média de $24^{\circ} \mathrm{C}$. Logo, a micose predomina na região Amazônica, em especial, no Brasil e na Colômbia (RIVITTI EA, 2018; BRITO AC e QUARESMA JAS, 2007). Além de casos em humanos, a infecção foi descrita em algumas espécies de golfinhos que vivem em estuários ou em cativeiro. Entretanto, até o momento, não foram descritos casos em botos, apesar de viverem em águas correntes do rio Amazonas, ou tucuxis, no rio Orinoco (TAVARES R, et al., 2011).

Acredita-se que se trata de um fungo saprófita do solo, da vegetação e da água. A forma de transmissão, apesar de destituída de comprovação, ocorre por inoculação do fungo por meio de soluções de continuidade da pele, como aquelas causadas por traumatismos diretos ou picadas de inseto. Da mesma forma, não há registros de transmissão entre humanos (RIVITTI EA, 2018; TAVARES R, et al., 2011; BRITO AC e QUARESMA JAS, 2007; ARENAS CM, et al., 2019). Além disso, a infecção tem predominância no sexo masculino, entre a faixa etária dos 20 a 45 anos, que exercem atividade no meio rural como agricultores, pescadores, seringueiros, mateiros e etc (SOUZA SP, et al., 2013).

Dados epidemiológicos esses que corroboram com o paciente-caso, o qual trabalhava como agricultor no momento provável da inoculação do fungo em região de mata, mais especificamente no município de Monte Alegre, estado do Pará, o qual faz parte da região Amazônica. 
Uma vez inoculado na derme, ocorre a fagocitose do fungo e inicia seu crescimento e multiplicação. A partir disso, pode ocorrer disseminação e proliferação micótica por via linfática ou por via hematogênica (TAVARES R, et al., 2011). No entanto, na grande maioria dos casos, a infecção limita-se à pele, principalmente, em áreas mais expostas como o pavilhão auricular e os membros inferiores. Raros são os casos da forma disseminada (BRITO AC e QUARESMA JAS, 2007).

Da mesma forma, a Doença de Jorge Lobo caracteriza-se pelo polimorfismo lesional, no qual a lesão inicial é uma pápula superficial ou até mesmo um nódulo que pode confluir e formar uma placa, a qual pode ser única ou múltipla. Dentre as lesões encontradas nessa infecção inclui máculas, pápulas, nódulos, gomas, placas nodulares, lesões verruciformes, úlceras e cicatrizes (ARENAS CM, et al., 2019; SOUZA SP, et al., 2013).

O paciente-caso apresentava, no primeiro atendimento, o tipo lesional mais comum dessa infecção (RIVITTI EA, 2018), uma lesão única com aspecto queloidiforme com bordas definidas e descamação furfurácea, localizada em região medial do joelho esquerdo e com parâmetros $4 \mathrm{~cm} \times 3,5 \mathrm{~cm}$. Negava prurido ou ardor. Apesar de esses últimos sintomas poderem compor o quadro clínico da Lobomicose, como foram descritos nos relatos de casos (BUSTAMANTE B, et al., 2013; SOUZA MN, et al., 2015; BELTRAME A, et al., 2017).

A resposta à infecção pelo fungo parece depender da suscetibilidade do hospedeiro ao agente etiológico (TAVARES R, et al., 2011). Logo, diferentes formas clínicas podem ser observadas como: infiltrativa ou indeterminada, queloidiana, gomosa, ulcerosa e verruciforme (BRITO AC e QUARESMA JAS, 2007; SOUZA SP, et al., 2013). Alguns estudos revelam que respostas imunes celulares prejudicadas sejam as responsáveis pela cronicidade das lesões e viabilizam a proliferação do fungo no interior dessas lesões. Dessa forma, a quantificação de citocinas no soro e em sobrenadantes de cultura de células de portadores da lobomicose revelou diminuição de IL-2 e aumento de IL-4 e IL-6, isso evidencia a predominância do perfil imunológico Th2, provocando alterações dos mecanismos responsáveis por conter o agente etiológico (RIVITTI EA, 2018).

Além disso, Xavier MB, et al. (2008) afirmam que os macrófagos infiltrados pelo agente micótico têm níveis elevados de fator de crescimento transformador- $\beta$, a qual é uma interleucina que suprime a atividade lítica dos macrófagos e, consequentemente, promove a produção de fibrose, esse arranjo contribui para a formação de lesões queloidianas. Essa interleucina é capaz ainda de inibir a expressão do óxido nítrico e interferon gama, o que pode comprometer diretamente a imunidade mediada por células; além de estimular a proliferação de linfócitos T CD8 e, consequentemente, promover fibrose (FRANCESCONI VA, et al., 2014).

Da mesma forma, outro estudo realizado por Quaresma JAS, et al. (2015) indica alterações na microvasculatura local, o que pode interferir diretamente no desenvolvimento de uma resposta imunológica eficaz mediada por células e isso pode justificar a restrição do agente etiológico no local da lesão na maioria dos casos.

O tempo de evolução da lesão do paciente-caso foi de três anos, o que confirma a cronicidade da infecção, apesar de a grande parte dos casos possuir tempo de evolução muito superior a essa, como relatado nos estudos clínicos de Miranda MFR, et al. (2011) com evolução de 10 anos; Furtado NA, et al. (2013), 25 anos; Souza MN, et al. (2015), 28 anos; e Moura Filho FR, et al. (2017), 28 anos. Embora possa haver acometimento de linfonodos regionais, não foi observado envolvimento linfonodal no paciente-caso. Além disso, a disseminação sistêmica é rara (PANIZ-MONDOLFI A, et al., 2012).

O diagnóstico clínico é um desafio, grande parte das vezes, devido a casos frequentemente atípicos e às lesões assemelharem a de outras patologias. Por isso a importância do conhecimento acerca do diagnóstico diferencial.

No início do quadro clínico, as lesões infiltradas do tipo placa podem ser confundidas com leishmaniose cutânea; assim como as lesões com aspecto queloidal típicas da lobomicose devem ser diferenciadas daquelas da hanseníase virshowiana; e as lesões com aspecto verrucoso, vegetativo ou nodular podem levantar a suspeita de esporotricose, cromoblastomicose, paracoccidioidomicose, quelóides ou processos neoplásicos (CHEURET M, et al., 2014). 
O diagnóstico laboratorial é feito por meio do exame micológico, anatomopatológico e imuno-histoquímico. É difícil obter a cultura do fungo devido ao longo período de crescimento do agente. O material analisado pelo exame micológico é decorrente do macerado de fragmento de biopsia cutânea, da escarificação, da raspagem ou do uso de fita-cola a partir da lesão cutânea.

O exame demonstra células leveduriformes esféricas de 6 a $12 \mu \mathrm{m}$ de diâmetro, com distribuição em rosário ou em cadeia de 3 a 9 elementos (TAVARES R, et al., 2011; ARAÚJO MG, et al., 2018). A característica diagnóstica do fungo Lacazia loboi é a cadeia de gomos do mesmo tamanho, isso difere de Paracoccidioides, no qual os gomos são menores que a célula mãe (CHEURET M, et al., 2014).

Ao exame anatomo-patológico a epiderme pode apresentar-se normal, atrófica, hiperplásica ou ulcerada. Por fim, a análise imuno-histoquímica revela positividade para o CD68 e forte imuno-marcação para TGF- $\alpha$ e IFN-y (ARAÚJO MG, et al., 2018).

O tratamento da Doença de Jorge Lobo é difícil. Até o momento, nenhum tratamento medicamentoso eficaz está disponível. As terapêuticas antifúngicas, utilizadas com sucesso em outras micoses profundas, não se mostraram eficazes na lobomicose (RIVITTI EA, 2018; ARENAS CM, et al., 2019).

Acredita-se que as características do fungo Lacazia loboi e a fibrose que se desenvolve em resposta a ele, em casos de longa data, sejam uma barreira para a ação de medicamentos antifúngicos (ARAÚJO MG, et al., 2018). Logo, o tratamento de escolha, em especial para lesões localizadas, é o cirúrgico, por meio da exérese, eletrocoagulação ou criocirurgia (BRITO AC, et al., 2007; SOUZA SP, et al., 2013).

O paciente-caso apresentava tratamento prévio, em outro nosocômio, com criocirurgia com nitrogênio líquido. No entanto, com recidiva da lesão e crescimento progressivo há cerca de um ano. Apesar de a literatura citar a criocirurgia como uma alternativa terapêutica, poucos são os relatos clínicos documentados que utilizaram essa opção de tratamento. Diferentemente do paciente em questão, Carneiro FP, et al. (2009) e Araújo MG, et al. (2018) relataram sucesso com o uso da criocirurgia, com regressão completa das lesões e sem sinais de recidiva. Embora esse último estudo também tenha utilizado associação com tratamento medicamentoso e com excisão cirúrgica.

Dentre as opções de tratamento, a exérese completa das lesões é a que demonstra resultados mais satisfatórios. Isso é evidenciado nos casos relatados por Miranda MFR, et al. (2011), Souza MN, et al. (2015), Beltrame A, et al. (2017) e Arenas CM, et al. (2019). Além disso, os três últimos estudos utilizaram tratamento medicamentoso concomitante.

Os resultados satisfatórios também foram alcançados no paciente-caso com a utilização desse tratamento, por meio da ressecção da lesão com margem de $4 \mathrm{~mm}$ e reconstrução com retalho de pele retirado da panturrilha. Não houve associação com medicamentos antifúngicos oral ou tópico.

O paciente está em acompanhamento médico há 18 meses, sem sinais de recidiva da lesão. Enquanto isso, a exérese parcial das lesões pode demonstrar recidivas, como relatado em um caso clínico (FURTADO NA, et al., 2013).

Entretanto, em lesões extensas ou disseminadas, pode haver recidivas com os métodos cirúrgicos devido à impossibilidade de ressecção total das lesões com margem de segurança. Nesses casos, portanto, a opção terapêutica passa a ser o tratamento medicamentoso, o qual pode ainda ser complementado pela excisão cirúrgica em série de algumas lesões (ARAÚJO MG, et al., 2018; TAVARES R, et al., 2011). O estudo de Bustamante B, et al. (2013) relatou um caso com resultados terapêuticos satisfatórios com a utilização do antifúngico posaconazol, administrado de forma prolongada e bem tolerado.

No entanto, uma das limitações do seu uso é o alto custo do medicamento, principalmente, levando em consideração que a epidemiologia da doença são pescadores, agricultores, mateiros e etc. Da mesma forma, houve relatos favoráveis com a utilização da clofazimina ou a associação de clofazimina e itraconazol, porém, com posterior recidiva das lesões. Outras tentativas medicamentosas com sulfas, cetoconazol, anfotericina B, flucitosina, dapsona, itraconazol, foram feitas com resultados insatisfatórios (RIVITTI EA, 2018; SOUZA SP, et al., 2013). 
Não há registros de morte associado à Doença de Jorge Lobo. No entanto, é uma patologia, muitas vezes, desfigurante e que pode, de certa forma, afetar a qualidade de vida do paciente (WOODS WJ, et al., 2010). Apesar de até o momento a literatura não apresentar uma terapia completamente eficaz contra o agente Lacazia loboi, até mesmo com a ressecção cirúrgica, possivelmente devido à ressecção insuficiente (ARAÚJO MG, et al., 2018), o paciente-caso apresentou resposta completamente satisfatória com a terapia empregada.

As falhas terapêuticas fundamentadas em ampla revisão de literatura e a raridade da doença motivaram a pesquisa referente ao tema e a descrição do caso assistido. Além disso, a lobomicose deve ser sugerida como diagnóstico diferencial em pacientes com lesões queloidianas na pele de áreas expostas, principalmente, se existe história de contato com áreas nas quais a doença é endêmica. Esta é uma doença deformante que, muitas vezes, afeta a qualidade de vida dos pacientes, e a cirurgia mostrou-se ser uma forma de tratamento efetiva para a retirada das lesões, especialmente se a doença for diagnosticada em uma fase precoce.

\section{REFERÊNCIAS}

1. ARAÚJO MG, et al. Lobomicose: um desafio terapêutico.Anais Brasileiros de Dermatologia, $2018 ; 93$ (2).

2. ARENAS CM, et al. Lobomycosis in Soldiers, Colombia. Emerging Infectious Diseases Journal, $2019 ; 25$ (4): $654-660$.

3. BELTRAME A, et al. Case Report: Molecular Confirmation of Lobomycosis in an Italian Traveler Acquired in the Amazon Region of Venezuela. The American Journal of Tropical Medicine and Hygiene, 2017;97 (6): 1757 - 1760.

4. BRITO AC, QUARESMA JAS. Lacaziose (doença de Jorge Lobo): revisão e atualização. Anais Brasileiros de Dermatologia, 2007; 82 (5).

5. BUSTAMANTE B, et al. Lobomycosis Successfully Treated with Posaconazole. The American Journal of Tropical Medicine and Hygiene, 2013; 88 (6): 1207 - 1208.

6. CARNEIRO FP, et al. Lobomycosis: diagnosis and management of relapsed and multifocal lesions. Diagnostic Microbiology and Infectious Disease, 2009;65 (1): 62-64.

7. CHEURET M, et al. A 43-Year-Old Brazilian Man With a Chronic Ulcerated Lesion. Clinical Infectious Diseases, 2014; 59 (2): 314-315.

8. FRANCESCONI VA, et al. Lobomycosis: epidemiology, clinical presentation, and management options. Ther Clin Risk Manag., 2014; 10: 851-860.

9. FURTADO AN, et al. Doença de Jorge Lobo:Relato de Caso e Revisão de Literatura.RevistaPatologia Tropical, 2013; $42(4): 459-467$.

10. KORTE RL, et al. Cirurgia plástica e o tratamento de doenças infectocontagiosas: Lobomicose. Rev. Bras. Cir. Plást., 2019; 34(1):163-172.

11. MIRANDA MFR, et al. Doença de Jorge Lobo de localização labial exclusiva. Anais Brasileiros de Dermatologia, 2011; 86(2).

12. MOURA FILHO FR, et al. Lobomicose. Revista SPDV, 2017; 75(3): 289-292.

13. PANIZ-MONDOLFI A, et al. Lobomycosis: an emerging disease in humans and delphinidae. Mycoses: diagnosis, therapy and prophylaxis of Fungal Diseases, 2012;55 (4): 298-309.

14. QUARESMA JAS, et al. Analysis of microvasculature phenotype and endothelial activation markers in skin lesions of lacaziosis (Lobomycosis). Microbial Pathogenesis, 2015;78 (1): 29-36.

15. RIVITTI EA. Micoses Profundas. Dermatologia de Sampaio e Rivitti. $4^{\circ}$ edição. São Paulo: ArtesMédicas, 2018, p. 759761.

16. SOUZA SP, et al. Doença de Jorge Lobo e a importância de seu estudo para a Patologia Animal e para a Patologia Humana. Revistalnterdisciplinar de EstudosExperimentais, 2013; 5 (1): 39-42.

17. SOUZA MN, et al. Lobomycosis of the Lower Limb in an Amazonian Patient. The American Journal of Tropical Medicine and Hygiene, 2015; 93 (4): $675-676$.

18. TAVARES R, et al. Lobomicose ou Doença de Jorge Lobo revisão da literatura.Rev. Portuguesa de DoençasInfecciosas, 2011; 7(3) 111-117.

19. WOODS WJ, et al. Dez anos de experiência com a doença de Jorge Lobo no estado do Acre, região amazônica, Brasil. Revista do Instituto de Medicina Tropical de São Paulo, 2010; 52 (5).

20. XAVIER MB, et al. Macrophage and TGF- $\beta$ immunohistochemical expression in Jorge Lobo's disease. Human Pathology, 2008;39 (2): 269-274. 